\title{
Instrumente in der \\ Unfallchirurgie, Orthopädie und Gynäkologie
}

Kirsten Wagenhäuser, Karl-Heinz Wagenhäuser

Im fünften Teil unserer Serie zeigen wir Ihnen einige Instrumente, die in der Unfallchirurgie, Orthopädie und Gynäkologie hauptsächlich zur Anwendung kommen. Typische fassende und schneidende Instrumente aus der Viszeralchirurgie haben wir bereits im letzten Artikel vorgestellt, diese werden natürlich in allen chirurgischen Bereichen in den unterschiedlichsten Ausführungen und Größen verwendet.
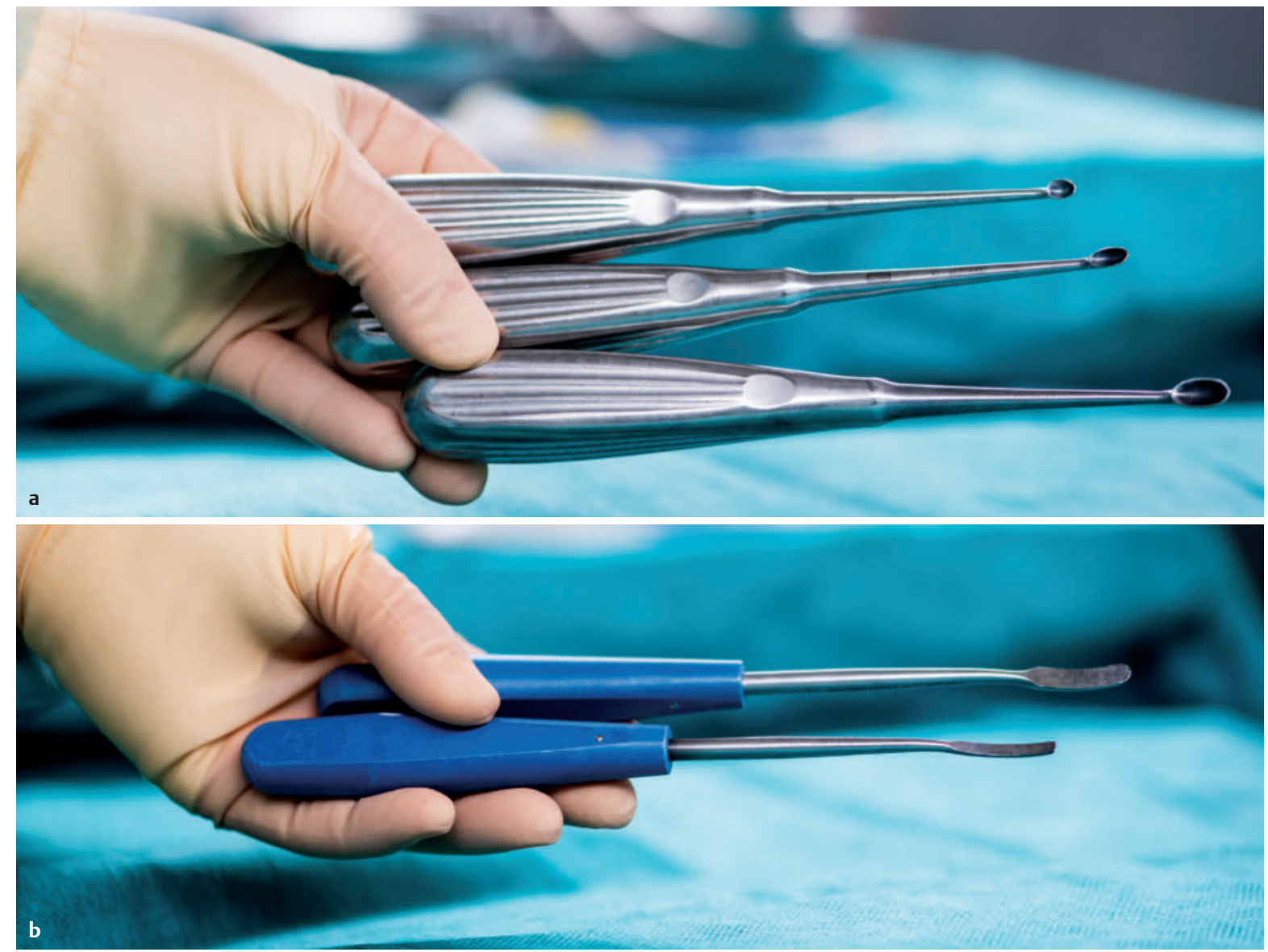

Abb. 1 (Quelle: Paavo Blåfield)

a Ein Instrument, das in allen chirurgischen Bereichen Gebrauch findet, ist der scharfe Löffel. Mit ihm kann der Chirurg Wunden an frischen Nekrosen abtragen und allgemein unerwünschtes Gewebe oberflächlich entfernen. Der Unfallchirurg kann mit sehr kleinen Löffeln Frakturspalten von kleinen Fragmenten säubern. Größere Löffel werden gerne zum Abschaben von unerwünschten Weichteilen am Knochen verwendet. b Zum Entfernen und Abschieben von Knochenhaut und Weichteilen am Knochen nehmen sowohl Unfallchirurgen als auch Orthopäden Raspatorien. Auf unserem Bild sind kleine Raspatorien abgebildet: Das untere wird als scharfes, das obere als stumpfes Raspatorium bezeichnet. 

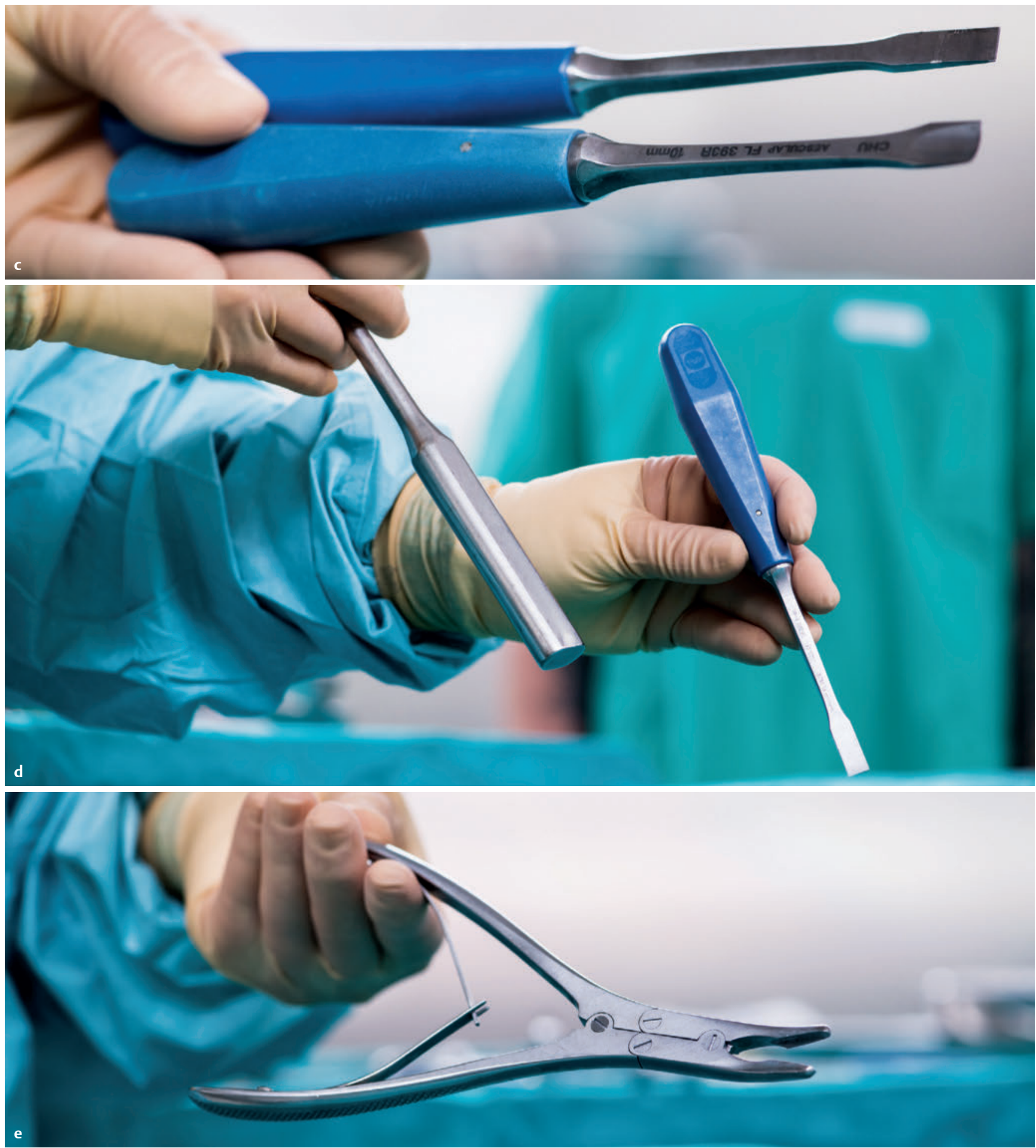

Abb. 1 Fortsetzung. (Quelle: Paavo Blåfield)

c Meißel gibt es im unfallchirurgischen OP in Hülle und Fülle. Unsere kleinsten Meißel haben eine Breite von ein bis zwei Millimetern, die größten Meißel in diesem Bereich sind bis zu 30 Millimeter breit. Grob kann man die Meißel in Hohl- und Flachmeißel unterteilen. Flachmeißel nimmt man zum Osteotomieren oder allgemein zum Entfernen von Knochenmaterial. Hohlmeißel kommen zum Einsatz, um überstehendes Knochenmaterial, wie zum Beispiel Osteophyten, zu entfernen. $\mathbf{d}$ Meißel werden normalerweise immer mit einem entsprechenden Hammer angereicht. Auf dem Bild ist ein kleiner Hammer zu sehen, der zirka 200 Gramm wiegt. Große Hammer können ein Gewicht von einem Kilogramm und mehr haben. Beim gleichzeitigen Anreichen von beiden Instrumenten hält man den Hammer am Hammerkopf, sodass der Anwender diesen am Stiel anfassen kann. Der Meißel wird zeitgleich in der Mitte am Schaft angereicht, um am Handgriff entgegengenommen werden zu können. So wird verhindert, dass sich jemand an den geschliffenen und scharfen Meißelklingen verletzt. e Die Hohlmeißelzange, meistens als Luer bezeichnet, hat eigentlich nichts mit einem Meißel zu tun. Sie ist eine scharfe Gewebe- beziehungsweise Knochenfasszange, deren Maul hohl und deren Fassbereiche scharf geschliffen sind. Mit ihr wird überschüssiges beziehungsweise unerwünschtes Knochengewebe grob abgetragen. Bei Wirbelsäulenoperationen werden mit dem Luer zum Beispiel Dornfortsätze entfernt. 

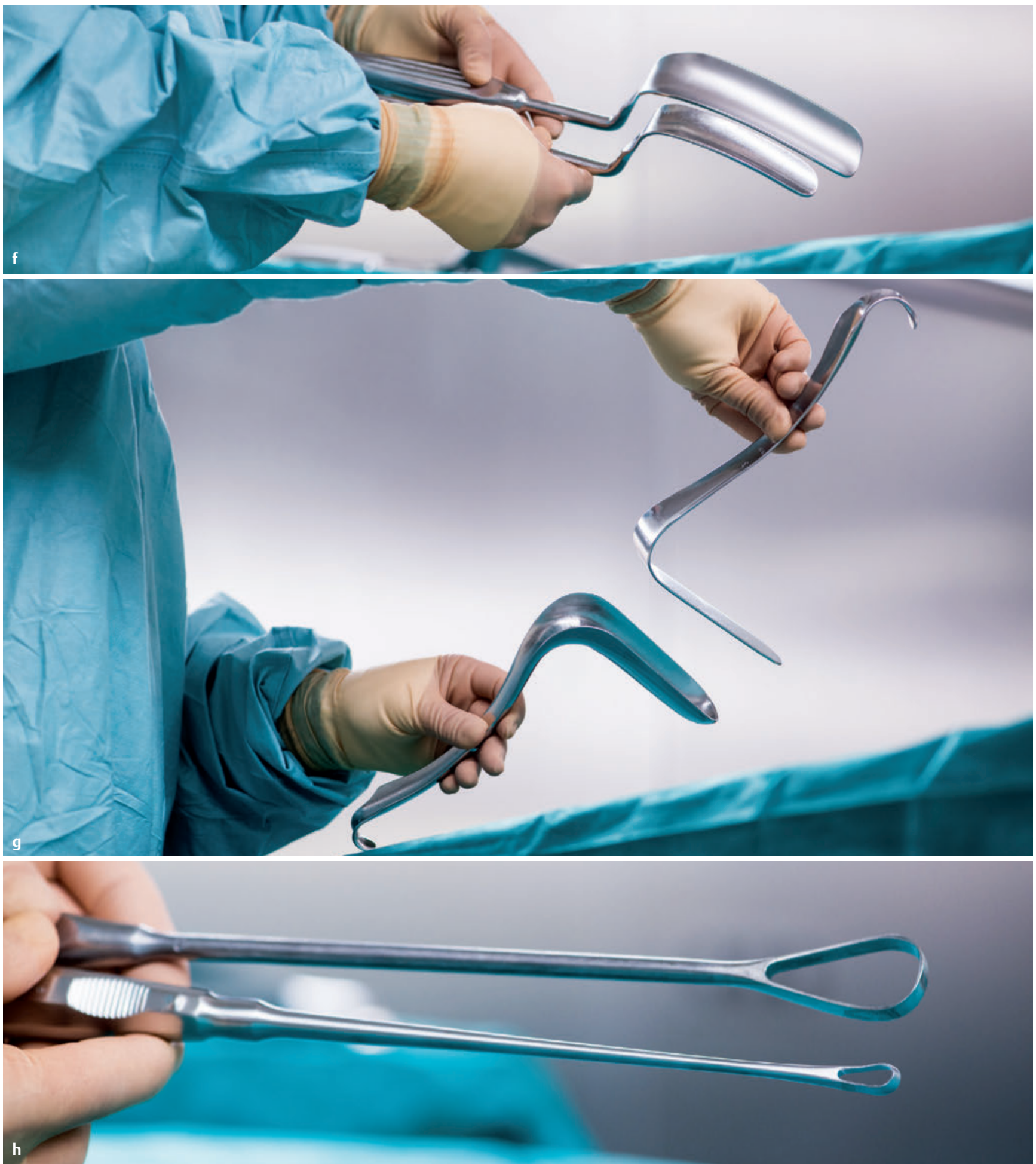

Abb. 1 Fortsetzung. (Quelle: Paavo Blåfield)

f Die Gynäkologie verwendet zum Weghalten von Gewebe in der Tiefe gerne Breiskys. Sie unterscheiden sich von den herkömmlichen Wundhaken - wie Langenbeck - durch einen geraden Handgriff und gekröpfte Haltefläche und werden häufig in der Vagina eingesetzt. Aber auch bei abdominalen Eingriffen werden sie oft von Gynäkologen verwendet, besonders wenn diese im Douglas operieren. g Zum Desinfizieren und Inspizieren der Vagina kommen unterschiedliche Spekula zum Einsatz. Auf dem Bild zeigen wir ein vorderes und ein hinteres Blatt eines Spekulums. Das hintere Blatt ist eigentlich ein Rinnenspekulum, es wird in der Vagina auf das Rektum aufgesetzt. Das vordere Blatt ist zum Weghalten des Blasenpfeilers. h In der Gynäkologie werden zwei Arten von Curetten unterschieden: Scharfe Curetten werden zum Ausschaben und Gewinnen von Gewebe aus der Cervix und dem Corpus des Uterus verwendet, um dieses histologisch untersuchen zu können. Mit stumpfen Curetten werden nach Aborten oder nach Geburten Placentareste ausgeschabt, wobei eine Verletzung der Uteruswand verhindert werden soll, da diese durch die Schwangerschaft leichter verletzt werden könnte. Im Bild ist die größere Curette stumpf und die kleinere Curette scharf. Üblicherweise sind diese auch durch deutliche Riffelungen oder einen erhabenen Punkt am Handgriff zu unterscheiden. 

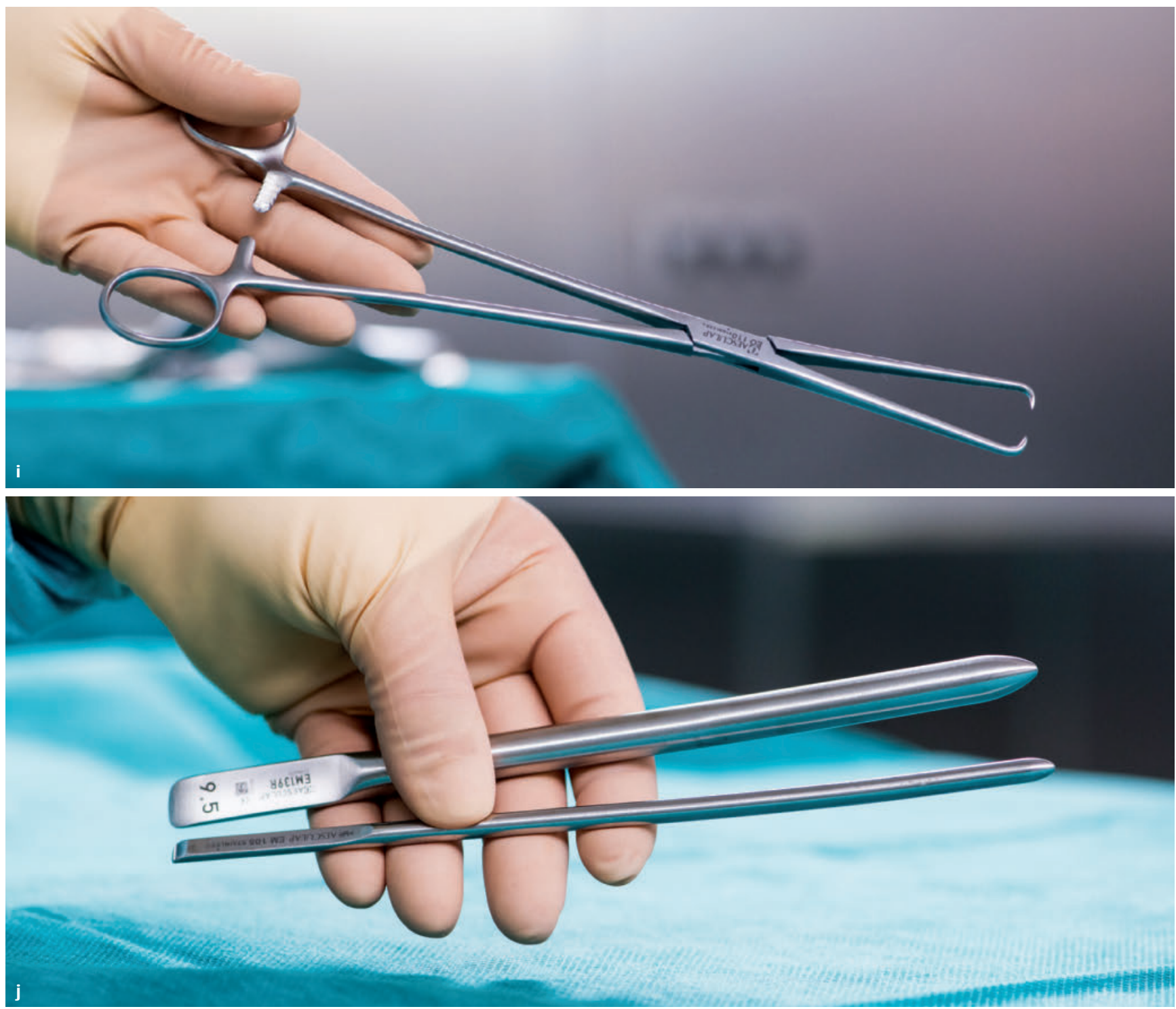

Abb. 1 Fortsetzung. (Quelle: Paavo Blåfield)

i Kugelklemmen sind einzinkige, lange Halteklemmen, die meistens zum Fassen der Portio verwendet werden. j Zum vorsichtigen Aufdilatieren der Cervix bei Curettagen oder Hysteroskopien werden Hegarstifte der Größe nach genommen, das heißt, dass der Muttermundhals in halben Millimeterschritten aufgeweitet wird, um mit Instrumenten in den Corpus zu gelangen.

\section{ONLINE-TUTORIAL}

Besuchen Sie auch unser Online-Tutorial zur Instrumentenkunde. Videos dazu finden Sie im Internet unter: www.thieme.de/instrumentenkunde.

\section{Bibliografie}

DOI https://doi.org/10.1055/a-0666-5738

Im OP 2018; 8: 230-233

(c) Georg Thieme Verlag KG Stuttgart · New York ISSN 1611-7905

\section{Autorinnen/Autoren}

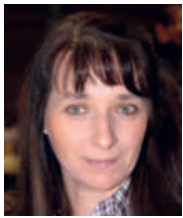

\section{Kirsten Wagenhäuser}

Praxisanleiterin, stellvertretende Bereichskoordinatorin Orthopädie, Unfallchirurgie, Hand- und Wiederherstellungschirurgie im ZOP des Leopoldina-Krankenhauses

Schweinfurt

E-Mail: kiwagenhaeuser@leopoldina.de

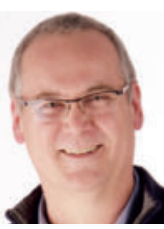

\section{Karl-Heinz Wagenhäuser}

Bereichskoordinator Gynäkologie am Leopoldina-Krankenhaus Schweinfurt E-Mail: khwagenhaeuser@leopoldina.de 